\title{
UTILITY OF CYSTICERCUS FASCIOLARIS ANTIGEN IN DOT ELISA FOR THE DIAGNOSIS OF NEUROCYSTICERCOSIS
}

\author{
NITIN SHUKLA, NUZHAT HUSAIN, GIRDHAR G AGARWAL', MAZHAR HUSAIN²
}

\section{ABSTRACT}

\begin{abstract}
BACKGROUND: Clinical diagnosis of neurocysticercosis (NC) is established by CT scan and MRI. However, absolute diagnosis is not possible in a fair number of cases, and serological assays are used as adjunct. Besides, CT scan and MR imaging are resourceintensive tests and not practical for screening in endemic areas. AIM: To provide a low cost, efficient, and reproducible assay for the detection of antibodies against cysticerci. Hence we have attempted to standardize and evaluate the diagnostic utility of the cysticercus fasciolaris antigen in a Dot ELISA assay for diagnosis of NC. SETTNG AND DESIGN: Tertiary hospital-based, case-control series. MATERIALS AND METHODS: Confirmed cases of NC diagnosed by presence of ring lesions in CT scan or M R imaging with presence of scolex were taken as positive controls $(n=50)$. Negative controls $(n$ $=50$ ) included subjects with normal CT scan studies $(n=30)$ and diseased controls with ring lesions in CT scan confirmed to be neurotuberculosis ( $n=20$ ). Dot ELISA was standardized and validated with commercially available ELISA (UBI, USA) using sera from the study groups. STATISTICAL ANALYSIS: Chi-square test was used to compare the immunodiagnostic performance of the two tests. $P$ value less than $.05(P<0.05)$ was considered significant. RESULTS: The Dot ELISA had a sensitivity of $88 \%$ and specificity of $74 \%$ with a positive predictive value of $77.19 \%$ and negative predictive value of $81.06 \%$. Likelihood ratios for a positive and a negative test were 3.4 and 0.2 . The sensitivity and specificity of commercial ELISA were $92 \%$ and $84 \%$ respectively. Difference between the performances of the two tests was not significant statistically. CONCLUSIONS: Dot ELISA has sensitivity and specificity comparable to ELISA for the diagnosis of $\mathrm{NC}$. The test is simpler, not requiring expertise and instrumentation. Further validation of the test as a screening tool is required.
\end{abstract}

Key words: Cysticercosis, Dot enzyme-linked immunosorbent assay (ELISA), ELISA

Departments of Pathology, 'Statistics, and

${ }^{2}$ Neurosurgery, King George's Medical University,

Lucknow, Uttar Pradesh, India

\section{Correspondence:}

Nuzhat Husain

Dept. of Pathology, King George's Medical University,

Lucknow - 226 003, India

E-mail: drnuzhathusain@hotmail.com

\section{INTRODUCTION}

Neurocysticercosis, an infestation caused by lodging of larval stage of Taenia solium in the brain, is the most common parasitic disease of the central nervous system. It is reported 
to cause 50,000 deaths per year throughout the world..$^{[1]}$ The disease is endemic in Andean area of South America, Brazil, China, Indian subcontinent, Indonesia, Mexico and Central America, Southeast Asia, and Sub-Saharan Africa. The diagnosis of neurocysticercosis is based on CT scan and MR imaging where the lesions are visualized as single or multiple ring-enhancing lesions. A set of diagnostic criteria have been listed with certain degrees of certainty by Del Brutto. ${ }^{[2]}$ Visualization of scolex of the parasite in cystic lesion is an absolute diagnostic criteria in CT scans or MR imaging. The scolex may not, however, be visualized in many cases due to varying levels of imaging. The differential diagnosis of cystic ring lesions in CT varies from inflammatory conditions like tuberculosis, toxoplasmosis, and brain abscess to small gliomas and arteriovenous malformations. Serological tests like ELISA and EITB are used as an adjunct to confirm the diagnosis.

Most immunodiagnostic tests utilize membrane or scolex antigens or their purified derivatives from cysticercus cellulosae, the human pathogen. ${ }^{[3,4]}$ We have used membrane antigen of cysticercus fasciolaris (larval stage of Taenia taeniaeformis pathogenic in rat) in anti-lgG and anti-IgM ELISA for the immunodiagnosis of NC. ${ }^{[5]}$ In the current study, we have designed and evaluated a Dot ELISA using membrane extract of cysticercus fasciolaris antigen for immunodiagnosis of neurocysticercosis.

\section{MATERIALS AND METHODS}

\section{Study setting}

Tertiary hospital-based study.

\section{Study design}

Case-control study for diagnostic test evaluation.

\section{Study sample}

Fifty confirmed cases of NC $(n=50)$ with ring-enhancing lesions in which scolex was visualized in CT scan or MR imaging were used as positive controls. Of these, 36 cases had a single cyst and 14 had multiple cysts in the parenchyma. Age- and sex-matched 50 negative controls included 30 cases with normal CT scan (undergoing the test for any other indication) and 20 diseased controls with $\mathrm{CT}$ ring lesions confirmed to be neurotuberculosis by MRI studies and MR spectroscopy. ${ }^{[6]}$ Cases with normal CT scan did not have any clinical evidence of cysticercosis in the form of epilepsy or subcutaneous nodules. Mean age of cases was $22.86 \pm 12.68$ years; and controls, 22.83 \pm 16.02 years. Four milliliters of blood was taken from the subjects of both the groups under aseptic precautions and allowed to clot. The test sera thus collected was aliquot and kept at $-70^{\circ} \mathrm{C}$ for future use. Both groups were screened for parasites in the stool examination. Institutional ethical clearance was obtained for the work, and all guidelines of animal ethics were followed. Prior consent of the subjects was taken, and all procedures followed were in accordance with the Helsinki Declaration of 1975 as revised in 2000 . $^{[7]}$

\section{Experimental techniques}

Preparation of antigen: The eggs from gravid segments of Taenia taeniaeformis which were obtained from intestines of cats were gently separated and suspended in normal saline at a concentration of 100 eggs $/ \mathrm{mL}$. Rattus rattus were orally infected with $0.2 \mathrm{~mL}$ of the egg suspension. After 60 days the rats were sacrificed, cysticercus fasciolaris cysts were dissected from the liver on ice, membranes were separated from the scolex and washed with cold phosphate buffered saline (PBS) at $\mathrm{pH}$ 7.4. The membranes thus separated were homogenized in five volumes of PBS, sonicated three times at $30 \mathrm{~S}$, and cold-centrifuged for 30 minutes at $20,000 \mathrm{~g}$. The concentration of protein was measured by Lowry's method. The supernatant at a protein concentration of 150 $\mathrm{mg} / \mathrm{dL}$ was used as the concentrated antigen.

Dot ELISA: One microliter of antigen (15 $\mu \mathrm{g} / \mathrm{mL}$ ) was dotted on to a square strip of nitrocellulose membrane $2 \times 2 \mathrm{~cm}$ in size. The strips were air-dried for 10 minutes. The strips were then blocked with $2 \%$ bovine serum albumin in PBS for 2 hours over shaker, followed by three washings with $0.2 \%$ Tween 20 in PBS $(\mathrm{pH} 7.4$ ) over a period of 15 minutes. All incubations were done in plastic ice trays. The strips were incubated at room temperature with test serum diluted (1:2000) in $0.2 \%$ Tween 20 in PBS (PBST), for 60 minutes on a shaker. Three washings were repeated. Rabbit antihuman IgG-peroxidase conjugate (Dakopatts, Denmark) was added at a dilution of 1:2000 in PBST and incubated for 30 minutes over the shaker and then washed thrice at the end. At the end, diaminobenzidine $(6 \mathrm{mg}$ in 5 $\mathrm{mL}$ of PBST with $30 \mu \mathrm{L}$ of hydrogen peroxide) was added. The strips were then incubated for 10 minutes in the dark. Reaction was stopped with distilled water. A deep brown-colored dot was considered as reactive, whereas no dot was considered as nonreactive. The serum samples were tested in a single-blind manner. The test was repeated on two different occasions to check for reproducibility, whic was $100 \%$. Blocked strips with antigen and other reagents were stored at $4^{\circ} \mathrm{C}$

ELISA: Commercial ELISA (UBI, USA) was used according to the manufacturer's instructions. The test detected presence of $\lg G$ antibodies to $T$. solium cysticerci.

\section{Statistical analysis}

Validation and consistency checks were carried out. Sensitivity, specificity, and predictive values and likelihood ratios for Dot ELISA and commercial ELISA in the diagnosis of neurocysticercosis were calculated. Chi-square test was used to compare the immunodiagnostic performance of the two tests. $P$ value less than 0.05 was considered significant.

\section{RESULTS}

\section{Dot ELISA}

Dot ELISA was positive in $44 / 50$ ( $88 \%$ ) radiologically proven NC cases and $13 / 50$ (26\%) negative controls (8/30 with normal CT scan and 5/20 with neurotuberculosis). Six radiologically proven cases that tested negative had single cysts. Overall the Dot ELISA was found to be $88 \%$ sensitive and $74 \%$ specific with a positive predictive value of $77.19 \%$ and negative predictive value of $81.06 \%$. Likelihood ratios were 3.4 for a positive and 0.2 for a negative test [Table 1].

\section{Commercial ELISA}

ELISA detected 46 of 50 positive controls, while $4 / 50$ could not be detected and were negative These four cases were also negative in the Dot ELISA. Forty-two negative controls wer test negative, while $8 / 50$ were test positive. 
Table 1: Performance characteristics of Dot ELISA for IgG and commercial enzyme-linked immunosorbent assay (ELISA) compared with CT scan (gold standard)

\begin{tabular}{lcccccc}
\multicolumn{1}{l}{ assay (ELISA) } & compared with & CT scan (gold standard) & & \\
\hline Test & $\begin{array}{c}\text { Sensitivity } \\
(95 \% \text { C.I.) }\end{array}$ & $\begin{array}{c}\text { Specificity } \\
(95 \% \text { C.l.) }\end{array}$ & $\begin{array}{c}\text { Positive predictive } \\
\text { value }(95 \% \text { C.I.) }\end{array}$ & $\begin{array}{c}\text { Negative predictive } \\
\text { value }(95 \% \text { C.I.) }\end{array}$ & $\begin{array}{c}\text { PositiveTest } \\
\text { Negative Test }\end{array}$ \\
\hline $\begin{array}{l}\text { Dot ELISA } \\
\text { Commercial }\end{array}$ & $88.0(79.0,97.0)$ & $74.0(62.0,86.0)$ & $77.2(66.0,88.0)$ & $81.6(76.0,96.0)$ & 3.4 & 0.2 \\
ELISA & $92.0(84.0,99.5)$ & $84.0(73.8,94.2)$ & $85.2(75.7,94.7)$ & $91.3(83.2,99.4)$ & 5.8 & 0.1 \\
\hline
\end{tabular}

Amongst the negative control group, 23/30 cases with negative CT and 19/20 of the diseased control group gave negative test. The sensitivity and specificity of the test were found to be $92 \%$ and $84 \%$ respectively [Table 1 ].

The differences between the values of sensitivity and specificity of the two tests were not found to be significant $(P$ value for sensitivity $=0.5 ; P$ value for specificity $=0.2$ ) [Table 1].

\section{DISCUSSION}

ELISA and EITB are the most commonly used serological tests for cysticercosis but require equipment and trained personnel and are not practical for use in screening in peripheral laboratories. Owing to the limitations of ELISA and EITB and problems faced in raising antigen in bigger mammals like pigs, there is a need for an easier, cheaper test format using alternate source of antigen. Hence the current study was undertaken to design and evaluate the efficacy of Dot ELISA using cysticercus fasciolaris antigen in immunodiagnosis of cysticercosis.

The cysticercus fasciolaris antigen, used in the study, can be produced in Rattus rattus under standardized conditions within a short duration of 60 days and can be a practical alternative to the commonly used cellulosae antigen requiring longer development time and evaluated diagnostic efficiency of experimental ELISA using cysticercus fasciolaris antigen and $\lg \mathrm{G}$ and $\lg \mathrm{M}$ antibodies and observed an overall sensitivity of $93.54 \%$ and a specificity of $84.2 \% .{ }^{[5]}$ However, the Dot ELISA is advantageous in terms of shorter test duration and the option of storage of the dotted antigen at room temperature for longer periods. Also, the test requires minimal infrastructure and reader and washer are required.

Dot ELISA has become increasingly popular for the diagnosis of many other parasitic diseases also, such as filariasis, ${ }^{[8]}$ malaria, ${ }^{[9]}$ and schistosomiasis, ${ }^{[10]}$ besides cysticercosis. Dot ELISA has been used earlier for diagnosis of cysticercosis using antigens derived from cysticercus cellulosae, ${ }^{[11-13]}$ cysticercus longicollis, ${ }^{[14]}$ and $T$. solium glycoproteins. ${ }^{[15]}$ Liu et al., 1996, have modified the Dot ELISA by Dot-immunogold silver staining for diagnosis of cysticercosis. ${ }^{[16]}$

We have found the Dot ELISA using cysticercus fasciolaris to be $88 \%$ sensitive and $74 \%$ specific. Though the commercial ELISA is $92 \%$ sensitive and $84 \%$ specific, the performance of Dot ELISA is comparable to it; and the differences between the values of sensitivity and specificity of the two tests are not statistically significant. Mandal et al. have maintenance of a bigger animal (pig). We have instrumentation unlike ELISA, where an ELISA also compared the performance of Dot Blot to ELISA, for the detection of antibodies against antigens from larval $T$. solium in children aged 5 to 12 years. Both tests gave similar sensitivity, viz., 89\%; while ELISA had better specificity, viz., $81 \%$, than Dot Blot (73\%). ${ }^{[17]}$ In another study by Biswas et al., Dot-ELISA was found to be $56.25 \%$ sensitive and $92 \%$ specific, as compared to plate ELISA, which was $43.75 \%$ sensitive and $98 \%$ specific. ${ }^{[12]}$ Jiang et al. have observed confirmed cases of neurocysticercosis showing $81.6 \%$ to $96.1 \%$ positive reactions in Dot-ELISA. ${ }^{[11]}$ It is reported that sensitivity and specificity of Dot ELISA generally vary from $56.25 \%$ to $97.61 \%$ and from $90.6 \%$ to $92 \%$ respectively. ${ }^{[11,12,14]}$ The difference can be explained by different criteria for patient selection and different gold standards used in different studies. We have used visibility of scolex in scans, an absolute diagnostic criterion, which also denotes active lesions; and hence seropositivity was high despite presence of a single cyst in several cases.

To detect any cross reactivity of intestinal parasites, a probable cause of false positives, the study group was subjected to stool test. We observed two cases with Ascaris and three cases with E. histolytica/E. dispar in stool. The sera of these cases did not cross react in Dot ELISA and commercial ELISA. No case of intestinal teniasis was encountered in the current study. Cross reactivity of the Cysticercus fasciolaris antigen has been evaluated in our earlier study evaluated in our earlier study, where we observed that the antigen does not cross react with cases of intestinal teniasis, hydatid disease, and Hymenolepis nana, which were included as diseased controls. ${ }^{[5]}$ The high false positivity in serological testing is a general diagnostic fallacy in regions with high endemicity fo cysticercosis due to persistence of antibodie following exposure. Further presence of cysts a other sites, including muscle and subcutaneous tissue, cannot be completely and convincingly ruled out in controls by clinical screening methods. These cysts can give an immunopositive result in serological tests, which is then erroneously categorized as false positive.

We conclude that Dot ELISA is a simple test with sensitivity comparable to ELISA. Cysticercus fasciolaris can be used as an alternative source of antigen in immunodiagnostic tests for the human pathogen. While the test may be used for screening for seropositivity in suspected cases, the localization and staging of the cyst can be done by radio-imaging techniques. The test needs to be evaluated as a screening procedure in population-based studies, where sensitivity and specificity may vary due to varying disease endemicity and presence of other parasitic infections.

\section{ACKNOWLEDGMENT}

Indian Council of Medical Research, New Delh India, for funding and support of the research.

\section{REFERENCES}

1. Gemmel M, Matyas Z, Pawlowsky Z, Soulsby E J. Guidelines for surveillance, prevention and contro of Taeniasis-cysticercosis. Geneva: World Health Organization; 1983.

2. Del Brutto OH, Rajshekhar V, White AC Jr, Tsang VC, Nash TE, Takayanagui OM, et al. Proposed diagnostic criteria for neurocysticercosis. Neurology 2001;57:177-83. 
3. Rosas N, Sotelo J, Nieto D. ELISA in the diagnosis of neurocysticercosis. Arch Neurol 1986;43:353-6.

4. Molinari JL, Garcia-Mendoza E, de la Garza Y, Ramirez JA, Sotelo J, Tato P. Discrimination between active and inactive neurocysticercosis by metacestode excretory-secretary antigens of Taenia solium in an enzyme-linked immunosorbent assay. Am J Trop Med Hyg 2002;66:777-81.

5. Husain N, Jyotsna, Bagchi M, Husain M, Mishra MK, Gupta S. Evaluation of Cysticercus fasciolaris antigen for the immunodiagnosis of neurocysticercosis. Neurol India 2001;49:375-9.

6. Gupta RK, Husain M, Vatsal DK, Kumar R, Chawla S, Husain N. Comparative evaluation of magnetization transfer MR imaging and in-vivo proton MR spectroscopy in brain tuberculomas. Magn Reson Imaging 2002;20:375-81.

7. Human D, Fluss SS. The world medical association's declaration of helsinki: Historical and contemporary perspectives Available from: http://www.wma.net/e/ethicsunit/pdf/ draft_historical_contemporary_perspectives.pdf. ${ }^{* * *}$ Provide date of citation ${ }^{* * *}$.

8. Zheng HJ, Fuhrman JA, Xu M, Cheng WF, Reddy MV, Piessens WF. Comparison of Dot-ELISA with Sandwich ELISA in detecting circulating antigen in patients with bancroftian filariasis. Chin Med J 1990;103:709-12.

9. Oprandy JJ, Long GW. Processing and microfiltration of mosquitoes for malaria antigen detection in a rapid dot immunobinding assay. $J$ Clin Microbiol 1990;28:1701-3.

10. Rabello AL, Garcia MM, Dias Neto E, Rocha RS, Katz N. Dot-dye-immunoassay and dot-ELISA for the serological differentiation of acute and chronic schistosomiasis mansoni using keyhole limpet haemocyanin as antigen. Trans R Soc Trop Med Hyg 1993;87:279-81.

11. Jiang HJ, Yang LX, Pang SH, Sun T, Xie SP, Meng JM, et al. Dot-ELISA in the diagnosis of neurocysticercosis. Zhongguo Ji Sheng Chong Xue Yu Ji Sheng Chong Bing Za Zhi 1990;8:207-9.

12. Biswas R, Parija SC, Narayan SK. Dot-ELISA for the diagnosis of Neurocysticercosis. Rev Inst Med Sao Paulo 2004;46:249-52.

13. Agudelo $P$, Botero D, Palacio LG. Evaluation of the ELISA method for diagnosis of human cysticercosis in an endemic region. Biomedica 2005;25:488-95.

14. Vaz AJ, Nakamura PM, Camargo ME, Camargo ED, Ferreira AW. Dot-ELISA for the detection of anti-Cysticercus cellulosae antibodies in cerebrospinal fluid using a new solid phase (resin-treated polyester fabric) and Cysticercus longicollis antigens. Rev Inst Med Trop Sao Paulo 1996;38:391-6.

15. Plancarte A, Fexas M, Flisser A. Reactivity in ELISA and Dot ELISA of purified GP24, an immunodominant antigen of Taenia solium, for the diagnosis of human neurocysticercosis. Int $\mathrm{J}$ Parasitol 1994;24:733-8.

16. Liu YS, Du WP, Wu ZX. Dot-immunogold-silver staining in the diagnosis of cysticercosis. Int $\mathrm{J}$ Parasitol 1996;26:127-9.

17. Mandal J, Singhi PD, Khandelwal N, Malla N. Evaluation of ELISA and Dot ELISAs for the serodiagnosis of neurocysticercosis, in children found to have single or multiple enhancing lesions

Source of Support: Indian Council of Medical Research, New Delhi, India, Conflict of Interest: None declared. 